\title{
Klinische und apparative Untersuchungstechniken des Schultergelenks
}

\author{
Uli Schmiddem, Christian Krettek, Rupert Meller
}

\section{Zusammenfassung}

Die Diagnostik von Pathologien rund um das Schultergelenk ist komplex. Um die Diagnose einer Schulterläsion zu stellen bzw. deren Verlauf zu beurteilen, ist eine reproduzierbare klinische Befunderhebung essenziell. Dazu muss der Untersucher unter einer Vielzahl von Untersuchungstechniken auswählen. Der vorliegende Artikel soll ein Beispiel für einen standardisierten Untersuchungsgang mit nach wissenschaftlichen Gesichtspunkten ausgewählten Untersuchungstechniken bieten.

\section{Diagnostic Procedures \\ for the Shoulder Joint (Clinical and Instrumental)}

The clinical evaluation of the shoulder joint is a challenging task. It is mandatory to evaluate the patient's history. The physical examination should consist of a defined portfolio of different tests that cover most pathologies. The article presents a standardised examination algorithm with a selected number of tests. The most common pathologies and the most suitable tests to detect them are presented in this article.

mit der höchsten Sensitivität (= Prozentsatz der richtigerweise als krank Erkannten) und Spezifität (= Prozent der richtigerweise als gesund Erkannten) angeführt. Nicht die Verwendung möglichst vieler Tests führt zum Ziel einer sicheren Diagnose: Murrell et al. konnten zeigen, dass die Kombination von 4 klinischen Zeichen ausreicht, um mit einer 98\%-Genauigkeit eine Rotatorenmanschettenläsion zu diagnostizieren [1].

lich ihrer Spezifität und Sensitivität durchleuchtet wurden.

Im Folgenden soll anhand der aktuellen Literatur ein Überblick über die Möglichkeiten der klinischen und apparativen Schulteruntersuchung gegeben werden. Anschließend wird ein standardisierter Untersuchungsablauf der Schulter dargestellt, der im klinischen Alltag mit einer Beschränkung auf wenige aussagekräftige Funktionstests mit einer hohen Treffsicherheit die häufigsten Pathologien abdeckt. Um eine Praxisnähe zu gewährleisten, werden in diesem Untersuchungsablauf bewusst nur die Tests

OP-JOURNAL 2013; 29: 6-14

(c) Georg Thieme Verlag KG Stuttgart · New York DOI http://dx.doi.org/10.1055/s-0032-1328427

\section{Klinische Diagnostik}

\section{Anamnese, Inspektion, Palpation}

Die allgemeine klinische Befunderhebung der Schulter gliedert sich in Anamnese, Inspektion und Palpation. Hierdurch kann die Genese der Schulterpathologie bereits wesentlich eingegrenzt und im weiteren Verlauf durch die klinische Untersuchung zielführend abgeklärt werden.

Zu Beginn empfiehlt sich das Einholen von grundlegenden Informationen wie dem Alter des Patienten, Begleiterkrankungen, vorangegangene Verletzungen sowie Beruf und Hobbys um das Anforderungsprofil zu definieren. Auch eine rheumatische Polyarthritis kann ihre Erstmanifestation im Schultergelenk haben. Ferner gilt es ein evtl. traumatisches Ereignis als Auslöser herauszuarbeiten bzw. auszuschließen. Zudem sollten der zeitliche Verlauf und die bisherige Therapie erfragt werden. Auch der tageszeitliche Verlauf kann Erkenntnisse über die Genese der Beschwerden bringen. Ein ausgeprägter Nachtschmerz ist bspw. häufig bei Rotatorenmanschettenläsionen zu beobachten. Das Hauptleiden des Patienten sollte klar herausgearbeitet werden, indem als führende Symptomatik eine Bewegungseinschränkung, ein Schmerz oder eher ein Kraftverlust definiert wird.

Die Schmerzlokalisation kann ebenfalls Aufschluss über die zugrunde liegende Genese erbringen. So projiziert sich ein Akromioklavikulargelenksschmerz meist zusätzlich in den lateralen Aspekt der Schulter [2,3], während sich ein durch eine Läsion der langen Bizepssehne verursachter Schmerz in der Regel in dem anterioren Bereich des proximalen $\mathrm{Hu}-$ merus widerspiegelt. Itoi et al. konnten dagegen aber auch zeigen, dass sich die Lokalisation der Rotatorenmanschettenläsionen nicht mit der Lokalisation der Schmerzen in Zusammenhang bringen lässt [4].

Bei der Inspektion gilt es beide Schultern im Seitenvergleich anzuschauen. Speziell bei traumatischer Beschwerdegenese kann hier oftmals bereits eine Blickdiagnose gestellt werden, bspw. bei einem relativen Klavikulahochstand im AC-Gelenk (ACG) verursacht durch eine Akromioklavikulargelenksverletzung oder bei einer dislozierten Klavikulafraktur mit Kranialisierung des medialen Frakturfragments durch den Zug des M. sternocleidomastoideus. Eine diffuse Schwellung und Hämatombildung am proximalen Oberarm mit evtl. begleitendem Senkungshämatom kann dagegen auf eine proximale Humerusfraktur oder eine traumatische Rotatorenmanschet- 

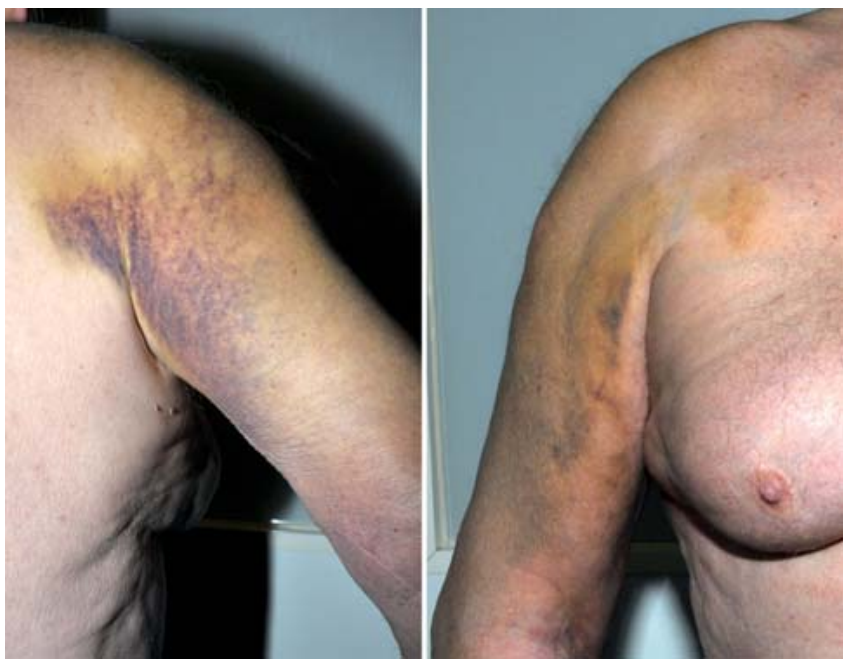

Abb. 1 Hämatom 1 Woche nach im MRT dokumentierter Massenruptur der Rotatorenmanschette.

tenläsion hindeuten (Abb. 1). Auch bei degenerativen Veränderungen kann die Inspektion die Diagnosestellung erheblich vereinfachen. Atrophien der Fossa supra- und/oder infraspinata können auf eine Rotatorenmanschettenruptur hinweisen. Auch eine Läsion des N. suprascapularis, bspw. durch ein Kompressionsphänomen im Verlauf des Nervs, kann eine höhergradige Atrophie bewirken (Abb. 2). Ebenso kann durch die Inspektion der dorsalen Schulterregion eine Asymmetrie im Bereich der Schulterblätter (z.B. Skapula alata) erkannt werden [5].

Bei der Palpation empfiehlt es sich, die Untersuchung von medial zu beginnen und sich nach lateral vorzuarbeiten. Es wird zuerst das Sternoklavikulargelenk (SCG) auf Stabilität und Schmerzhaftigkeit untersucht und anschließend die gesamte Klavikula bis zum ACG palpiert. Ein druck- und/oder translationsschmerzhaftes ACG ist ein klassisches Symptom einer symptomatischen ACG-Arthrose. Anschließend werden der Processus coracoideus, Tuberculum majus und minus sowie die lange Bizepssehne in der Region des Sulcus intertubercularis palpiert.

Ein Blick über die Grenzen des Schultergelenks hinaus sollte routinemäßig erfolgen: eine Untersuchung der Halswirbelsäule sowie ein orientierender neurologischer Status der oberen Extremität sind dazu erforderlich.

\section{Spezielle klinische Untersuchung}

Die weiterführende klinische Untersuchung beginnt nun mit einer orientierenden Untersuchung der Halswirbelsäule, um einen evtl. fortgeleiteten Schmerz zu erfassen. Die darauf folgende aktive und passive Beweglichkeitsuntersuchung der Schulter erfolgt stets im Seitenvergleich und wird für Flexion/Extension, Abduktion/Adduktion und Innenrotation/Außenrotation in Neutral- und $90^{\circ}$-Abduktionsstellung nach der Neutral-Null-Methode dokumentiert. Die Werte werden für den aktiven und den passiven Bewegungsumfang erhoben. Studien konnten zeigen, dass rein visuell vorgenommene Messungen nach der Neutral-Null-Methode sehr ungenau sind und einer hohen Interobserver-Varianz unterliegen [6]. Die Verwendung eines Goniometers ist daher als obligat anzusehen. Bei Verwendung eines Goniometers sind bei wiederholten Messungen lediglich Abweichungen von $8-10^{\circ}$ zu erwarten [7].

Im klinischen Alltag hat es sich bewährt, die Innenrotation durch die erreichbare Struktur am dorsalen Körperstamm (z.B. Th 12) zu messen („Schürzengriff“) und die Außenrotation durch den Griff an den Hinterkopf („Nackengriff“). Schürzen- und Nackengriff werden im Constant Score dazu verwendet, die Innenrotation und die Außenrotation im Schultergelenk abzuschätzen [8].

Die Frozen Shoulder ist als eine besondere Pathologie bei der Beweglichkeitsuntersuchung der Schulter zu erwähnen. Bei der Frozen Shoulder ist die Beweglichkeit in allen Ebenen eingeschränkt. Es wird zwischen einer primären Form mit unklarer Ätiologie und einer sekundären Form, die posttraumatisch oder postoperativ auftritt, unterschieden [9]. Klinische Kriterien sind insbesondere eine schmerzhaft eingeschränkte Elevation und Außenrotation sowie eine Einschränkung der aktiven und passiven Beweglichkeit. Je nach Stadium der Erkrankung (Stadium I-III) ist die Bewe-

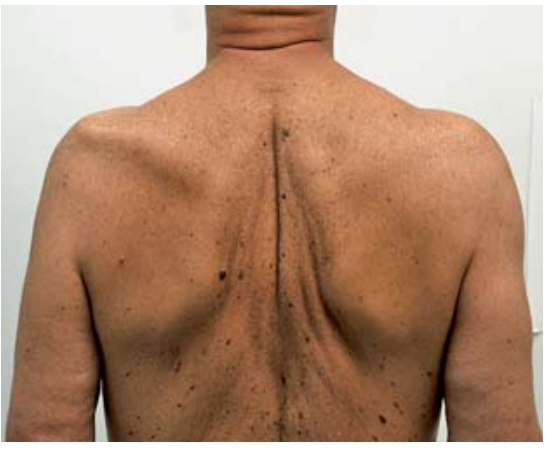

Abb. 2 Atrophie M. supra- und infraspinatus links.

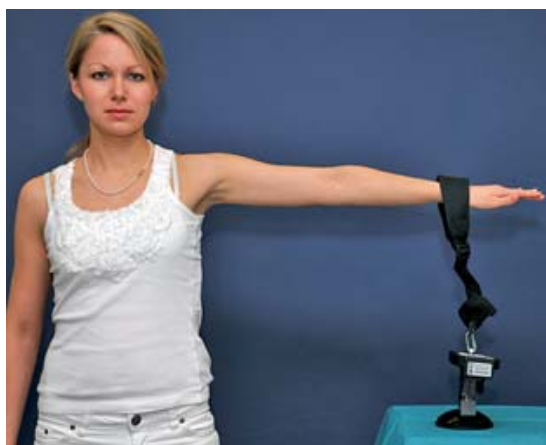

Abb. 3 Kraftmessung mit dem Dynamometer bei $90^{\circ}$ abduziertem Arm.

gungseinschränkung stärker oder weniger stark ausgeprägt [10].

\section{Funktionsbeurteilung und -dokumentation}

Sowohl bei der initialen Untersuchung als dann auch bei den jeweiligen Verlaufskontrollen sollte eine Funktionsbeurteilung und -dokumentation der Schulter mittels dafür vorgesehener Scoring-Systeme erfolgen. Diesbezüglich wurde eine Anzahl an Bewertungsuntersuchungen publiziert, wie z. B. das UCLARating-System, welches die Parameter Schmerz, Funktionseinschränkung, Bewegungsausmaß, Kraft und Gesamtzufriedenheit des Patienten erfasst [11]. Zudem gibt es den DASH-(Disabilities of the Arm-Shoulder-Hand)Score, der zu den ausschließlich subjektiven Scores zählt, und sich eher für die Verlaufsbeurteilung von distalen Radius- und Handfrakturen durchgesetzt hat [11]. Ebenfalls zur Selbsteinschätzung der allgemeinen Schulterfunktion durch den Patienten dient der Simple-ShoulderTest [11]. Der Patient wird gebeten, 12 standardisierte Fragen mit "Ja" oder „Nein“ zu beantworten. Je mehr Fragen mit „Ja“ beantwortet werden können, desto besser stellt sich die Schulterfunktion des Patienten dar. 


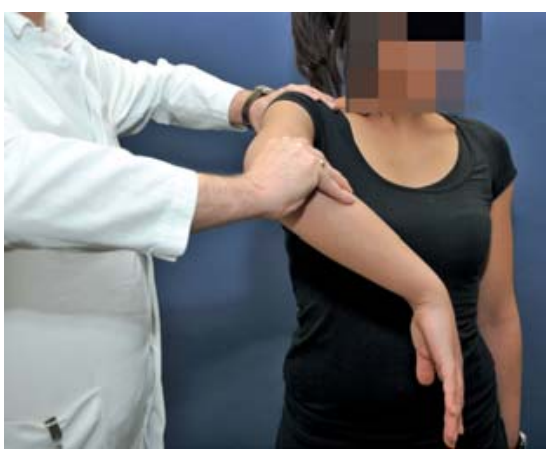

Abb. 4 Hawkins-Kennedy-Test.

In der eigenen Abteilung wird routinemäßig der Constant-Murley Score und die subjektive Schulterfunktion erfasst, bei Schulterinstabilitäten zudem der Rowe Score. Der Constant-Murley Score ist ein anerkannter und valider Score zur Beurteilung der Schulterfunktion, der die Parameter Schmerz, Aktivitäten des alltäglichen Lebens, Bewegungsumfang und Kraft erfasst [8]. Zur Beurteilung der Kraft verwenden wir ein Dynamometer (Abb. 3). Der Score wird bilateral erhoben, und es kann eine Anpassung an Alter und Geschlecht erfolgen. Der Constant-Murley Score eignet sich insbesondere zur Funktionsbeurteilung bei Rotatorenmanschettenläsionen, Arthrose des Schultergelenks und zur Verlaufsbeurteilung nach proximalen $\mathrm{Hu}-$ merusfrakturen. Bei der Beurteilung von Schultergelenksinstabilitäten wird die Funktionseinschränkung des Patienten jedoch nicht ausreichend dargestellt [12]. Daher verwenden wir bei Schulterinstabilitäten den Rowe Score, der die Parameter Funktion, Stabilität, Schmerz und Beweglichkeit einbezieht.

Es hat sich als sinnvoll erwiesen, bei jedem Patienten in der Schultersprechstunde die subjektive Schulterfunktion (SSV) zu erfassen. Der Patient wird dazu aufgefordert, die Schulterfunktion der verletzten Schulter im Vergleich zu einer vollständig normalen Schulter in Prozent anzugeben. Gilbart et al. konnten zeigen, dass eine gute Korrelation zwischen dem Constant-Murley Score und der SSV besteht. Zwar kann die SSV den Constant Score nicht ersetzen, ermöglicht aber eine einfache „Messung“ der Schulterfunktion [13].

\section{Impingementsyndrome}

Es wird zwischen dem subakromialen und subkorakoidalen sowie dem glenohumeralen Impingement unterschieden. Nach Neer unterscheidet man das subakromiale Impingement in ein primäres

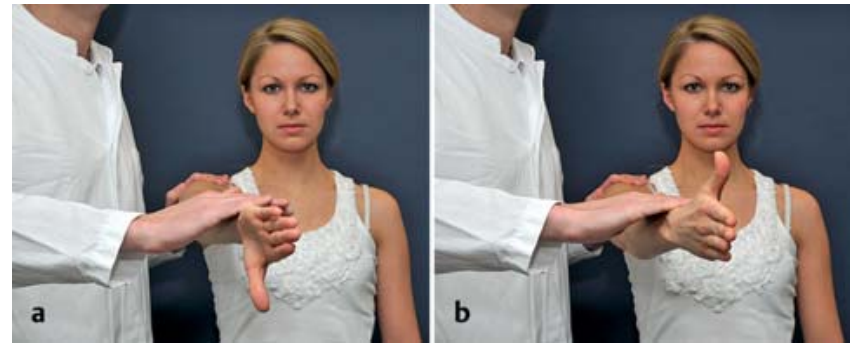

Abb. 5 O'Brien-Test (Active-CompressionTest). a Bei $90^{\circ} \mathrm{Fle-}$ xion, $10^{\circ}$ Adduktion und maximaler Innenrotation sowie Druck von kranial werden Schmerzen ausgelöst. b Bei Außenrotation hingegen lassen die Schmerzen nach.

und ein sekundäres Impingement. Das primäre Impingement entsteht durch eine mechanische subakromiale Raumeinengung, z.B. durch einen Akromionsporn oder posttraumatische Fehlheilungen mit Varusfehlstellung des Kopffragments mit konsekutiver Reizung des M. supraspinatus. Beim sekundären Impingement kommt es dagegen zu einer relativen Enge des Subakromialraums durch eine Volumenzunahme der Bursa oder der Rotatorenmanschette. Dementsprechend sind Impingementsyndrome unspezifische klinische Zeichen und als Symptom und nicht als Diagnose zu verstehen.

Das wohl bekannteste Impingementzeichen der Schulter ist der „Schmerzhafte Bogen“" (painful arc), bei dem zwischen etwa $60^{\circ}$ und $120^{\circ}$ aktiver Abduktion Schmerzen im Schultergelenk ausgelöst werden. Studienabhängig weist dieser Test eine Sensitivität von 67-75\% und eine Spezifität von $67-80 \%$ auf $[14,15]$.

Beim Impingementzeichen nach Neer führt der Untersucher den innenrotierten Arm des Patienten unter Fixation der Skapula in die Elevation und provoziert auf diese Weise einen subakromialen Konflikt zwischen Tuberculum majus und Schulterdach. Zur Verifizierung einer subakromialen Schmerzgenese ist dieser Test nach subakromialer Injektion mit einem Lokalanästhetikum nicht mehr schmerzhaft. Dieser indirekte Nachweis wird als Neer-Test bezeichnet und eignet sich somit als prognostischer Indikator für den Erfolg einer subakromialen Dekompression. Für das Impingement Zeichen nach Neer findet sich in der Literatur eine studienabhängige Sensitivität von 54-81\% und eine Spezifität von 30-95\% [14-16]. Beim Hawkins-Kennedy-Zeichen fixiert die körpernahe Hand des Untersuchers die Skapula (Abb.4). Mit der anderen Hand wird der in $90^{\circ}$ Abduktion und im Ellenbogengelenk $90^{\circ}$ flektierte Arm forciert innenrotiert. Der Test gilt als positiv bei Provokation von Schmerzen im Schultergelenk

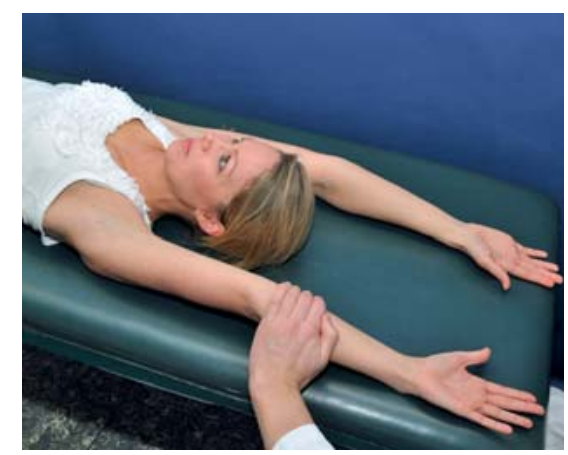

Abb. 6 Supine-Flexion-Resistance-Test.

(Abb.4). Für das Hawkins-Kennedy-Zeichen findet sich eine Sensitivität von $63-74 \%$ und eine Spezifität von 40-89\% [14-17]. Park et al. konnten nachweisen, dass bei Kombination eines positiven schmerzhaften Bogens, eines positiven Neer-Zeichens und eines positiven Hawkins-Zeichens eine 95\%-Vorhersagewahrscheinlichkeit für das Vorliegen einer Bursitis subacromialis oder eine Partial- oder Komplettruptur der Rotatorenmanschette besteht [18].

\section{Pathologie Bizepssehnenanker}

Verletzungen des Bizepssehnenankers/ SLAP-Läsionen (Superior-Labrum-Anterior-Posterior-Läsion) sind typischerweise traumatisch bedingt. Stürze auf den ausgestreckten, leicht flektierten und abduzierten Arm sowie Außenrotations-Abduktionstraumata sind typisch. Auch rezidivierende Mikrotraumata wie z.B. bei Überkopfsportarten werden in der Literatur genannt.

Zur klinischen Diagnostik einer SLAP-Läsion bewährt hat sich der O'Brien-Test (Active-Compression-Test) [19]. Der zu untersuchende Arm wird in $90^{\circ}$ Flexion, $10^{\circ}$ Adduktion und maximale Innenrotation (Daumen runter) gebracht (Abb. 5a). Der Untersucher versucht gegen den Widerstand des Patienten kraniokaudal gerichteten Druck auf den Arm auszuüben. Anschließend wird der gleiche Vorgang in Außenrotationsstel- 
lung (Daumen hoch) wiederholt (Abb. 5b). Der Test ist positiv, wenn bei Innenrotation Schmerzen in der Tiefe des Schultergelenks provoziert werden, die in Außenrotationsstellung nicht reproduzierbar sind. In der Literatur wird eine Sensitivität von 53-94\% und eine Spezifität von 28-92\% angegeben [20, 21]. Da sich dieser Test auch zur Diagnostik von ACG-Pathologien eignet, ist es bei positivem Testbefund entscheidend, die Schmerzlokalisation detailliert zu hinterfragen. Nur wenn der Schmerz sich in die Tiefe des Schultergelenks projiziert, gilt er als positiv hinsichtlich einer möglichen SLAP-Läsion. Ein weiterer Test zur Detektion einer SLAP-Läsion ist der „Supine-Flexion-ResistanceTest“. Der Patient liegt auf dem Rücken und legt die Arme in maximaler Flexionsstellung über den Kopf, die Handflächen zeigen deckenwärts. Der Patient versucht nun gegen den Widerstand des Untersuchers den Arm gestreckt anzuheben (Abb.6). Der Test gilt als positiv, wenn der Patient bei der Anspannung Schmerzen in der Tiefe des Schultergelenks angibt. Eine Studie von Ebinger et al. konnte für diesen Test im Vergleich zum O'Brien-Test eine zwar niedrigere Sensitivität, aber höhere Spezifität nachweisen [20]. Er eignet sich daher bei positivem O'Brien-Test zur weiteren Verifizierung der Verdachtsdiagnose.

Als apparative Bildgebung eignet sich am ehesten die MRT. Die MR-Arthrografie ist dabei der nativen MRT und der CT-Arthrografie überlegen $[22,23]$.

\section{Pathologien der langen Bizepssehne}

Rupturen der langen Bizepssehne fallen durch einen distalisierten Muskelbauch und eine nicht tastbare lange Bizepssehne (LBS) im Sulcus intertubercularis auf. Eine bei Palpation schmerzhafte Bizepssehne erbringt den Hinweis auf eine Pathologie und ist in ihrer studienabhängigen Sensitivität und Spezifität (53-57\% und 54-74\%) den klassischen Bizepssehnentests (Speed-, Yergason-Test) nahezu gleichwertig [24]. Aufgrund dieser niedrigen Werte empfiehlt eine aktuelle Publikation von Gill et al., sich nicht nur auf die körperliche Untersuchung zu verlassen, sondern empfiehlt bei klinischem Verdacht eine großzügige Indikationsstellung zur apparativen Diagnostik [24]. Eine bei Rotationsbewegungen im Sulcus intertubercularis „springende“ Bizepssehne ist dagegen wegweisend für eine Instabilität der LBS. Zur Abschätzung einer Affektion der LBS wird der Speed-
Test durchgeführt: es erfolgt eine aktive Flexion des supinierten Armes gegen Widerstand des Untersuchers (Abb. 7). Bei Läsionen der Rotatorenmanschetten kann der Test ebenso positiv sein. Sensitivität 54-71\%, Spezifität 67-81\% [25].

Als apparative Diagnostik wird die MRT empfohlen. Bei proximalen Bizepssehnenrupturen und Bizepssehnentendinopathien konnte dafür eine studienabhängige Sensitivität von 59-82\% und Spezifität von 64-88\% nachgewiesen werden [26]. Je nach Untersucher kann auch eine Sonografie aussagekräftig sein. Mit einem hochauflösenden Ultraschallgerät ist eine Abbildung der Stumpfenden bei Ruptur prinzipiell möglich, ebenso kann in der dynamischen Untersuchung eine Instabilität der LBS dargestellt werden.

\section{Rotatorenmanschettenläsionen}

Die Prävalenz einer kompletten Rotatorenmanschettenruptur bei über 70-jährigen Patienten liegt bei $20-30 \%$. Der überwiegende Anteil davon ist allerdings symptomlos [27]. Körperlich schwer arbeitende Menschen sind häufiger betroffen, ebenso erleiden Raucher häufiger eine Rotatorenmanschettendegeneration. Nach einer Publikation von Yamaguchi et al. sind Patienten mit symptomatischen Rotatorenmanschettenläsionen im Mittel 59 Jahre alt [28].

Die Supraspinatussehne ist gegenüber den anderen Sehnen der Rotatorenmanschette sowohl bei Total- als auch bei Partialrupturen mit Abstand am häufigsten betroffen. Bei degenerativ bedingten Defekten ist die Supraspinatussehne in ca. 95\% der Fälle beteiligt, etwa die Hälfte aller symptomatischen Defekte ist auf die Supraspinatussehne beschränkt. Die Infraspinatussehne ist in ca. 35\% und die Subskapularissehne in ca. $10 \%$ der Fälle beteiligt $[27,29]$.

$\mathrm{Zu}$ unterscheiden sind traumatische und degenerative Läsionen. Während traumatische Läsionen in der Regel akut mit Schmerzen und gleichzeitigem Kraftverlust einhergehen, stellen sich degenerative Läsionen initial eher ausschließlich mit Schmerzen dar. Hier kommt es im Verlauf schleichend zu einem zunehmenden Kraftverlust. Klinisch präsentieren sich kleinere Läsionen aufgrund der geringen biomechanischen Wirkung eher mit erheblichen Schmerzen und einer schmerzbedingten Funktionsminderung, während Massenrupturen in al- ler Regel weniger schmerzhaft sind, dafür aber mit einem Kraftverlust einhergehen [30]. Typisch für Rotatorenmanschettenläsionen sind ein ausgeprägter Nachtschmerz und ein spontaner Beschwerdebeginn.

Das Schultergelenk ist ein muskuloligamentär geführtes Gelenk. Hierdurch erklärt sich, dass es bei Vorliegen einer signifikanten Insuffizienz der Rotatorenmanschette $\mathrm{zu}$ einer Inkongruenz der Gelenkzentrierung kommt. Die Folge kann eine Migration des Kopfes mit Kranialisierung im Glenohumeralgelenk und das klinische Bild eines subakromialen Impingements sein. Folglich fallen die klassischen Impingementuntersuchungen (Painful Arc, Neer-Test, Hawkins-Test) positiv aus, sind allerdings als unspezifisch zu werten (hohe Sensitivität, niedrige Spezifität [18]). Der Hawkins-Test zeigt sich dabei mit einer studienabhängigen Sensitivität von 72-86\% und Spezifität von 45-67\% dem NeerTest und dem Painful Arc-Test überlegen [25].

In der Literatur findet sich eine große Anzahl an klinischen Tests, die zur Untersuchung der Rotatorenmanschette publiziert wurden. Im Folgenden werden verschiedene Tests gegenübergestellt und hinsichtlich ihrer Sensitivität und Spezifität beurteilt. Itoi et al. konnten belegen, dass sich die Aussagekraft eines Tests bei Kraftverlust gegenüber alleiniger Schmerzangabe signifikant erhöht [4].

Nicht die Verwendung möglichst vieler Tests führt zum Ziel einer sicheren Diagnose: Murrell et al. konnten zeigen, dass die Kombination von 4 klinischen Zeichen (Schmerz, Schwäche Abduktion, Schwäche Außenrotation und Impingement) ausreicht, um mit einer $98 \%$-Genauigkeit eine Rotatorenmanschettenläsion zu diagnostizieren [1]

\section{M. supraspinatus}

Zur Beurteilung der Sehne des M. supraspinatus (SSP) findet der Jobe-Test weite Verbreitung. Er kann als „empty-can“und „full-can“-Test durchgeführt werden. Der gestreckte Arm des Patienten wird $90^{\circ}$ abduziert und $30^{\circ}$ horizontal flektiert. Bei dem Empty-Can-Test wird eine Innenrotation des Armes durchgeführt (Daumen runter), dadurch werden die posterioren Aspekte der SSP getestet (Abb. 8a). Bei dem Full-Can-Test dagegen wird eine Außenrotation des 


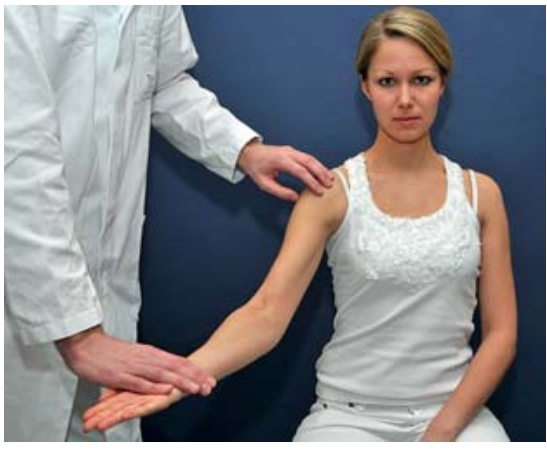

Abb. 7 Speed-Test.

Armes durchgeführt (Daumen hoch), wodurch die ventralen Aspekte des SSP getestet werden (Abb. 8b). Der Test ist positiv, wenn nach von kranial nach kaudal gerichtetem Druck des Untersuchers auf den Patientenarm ein Schmerz und/ oder ein Kraftverlust im Seitenvergleich eintritt [31]. Itoi et al. konnten für den Full-Can-Test eine Sensitivität von $80 \%$ und eine Spezifität von $50 \%$ zeigen, wenn die Auslösung von Schmerz als positives Testergebnis herangezogen wurde. Die Genauigkeit konnte gesteigert werden, wenn zusätzlich zu den Schmerzen ein Kraftverlust als positives Testergebnis gewertet wurde. Ähnliche Werte ergaben sich für den Empty-CanTest (Sensitivität 78\%, Spezifität 40\%). Auch hier konnte die Genauigkeit durch Beurteilung des Kraftverlusts erhöht werden [4]. Andere Publikationen zeigten vergleichbare Ergebnisse mit etwas höherer Spezifität, wenn die Auslösung von Schmerz und ein Kraftverlust einhergehend beobachtet wurden [32].

Der Drop-Arm-Test ist positiv, wenn der Arm nicht in der passiv erreichten $90^{\circ}$ Abduktionsstellung gehalten werden kann, seine Spezifität in Bezug auf eine SSP-Läsion ist mit 93\% sehr hoch. Allerdings ist die Sensitivität mit $10 \%$ zu gering, um den Test routinemäßig zum Ausschluss einer SSP-Verletzung heranzuziehen [1]. Ebenfalls zur Beurteilung der SSP-Funktion herangezogen wird der Starter-Test oder $\mathbf{0}^{\circ}$-Abduktionstest. Hier wird der Patient aufgefordert, die am Körper in Neutralstellung herabhängenden Arme gegen die Kraft des Untersuchers aktiv zu abduzieren. Bezüglich der Aussagekraft dieses Tests fehlen klinische Studien in der Literatur.

An apparativer Diagnostik werden routinemäßig konventionelle Röntgenaufnahmen der Schulter in 3 Ebenen mit True a.-p., axialer und Outlet-Aufnahme durchgeführt. Bei klinischem Verdacht

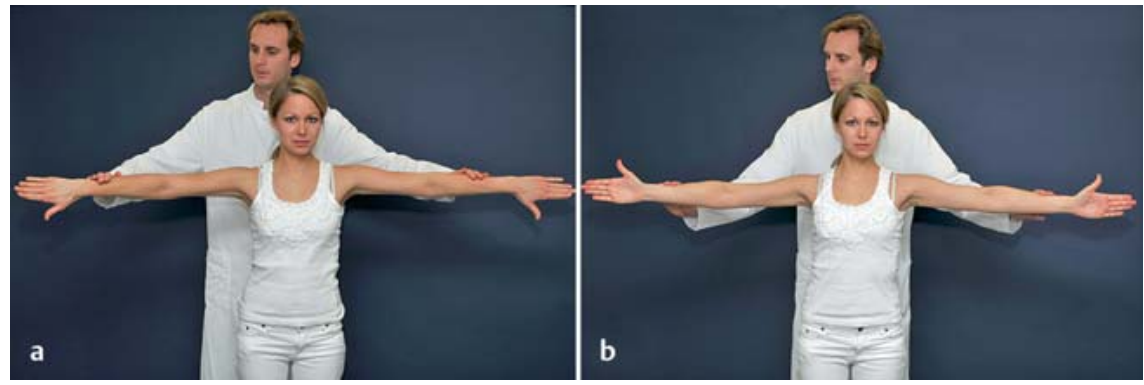

Abb. 8 Jobe-Test. a Daumen zeigen bei Innenrotation nach unten (empty can), es werden die eher dorsal gelegenen Teile der Rotatorenmanschette getestet. b Daumen zeigen bei Außenrotation nach oben (full can), es werden die eher ventral gelegenen Teile der Rotatorenmanschette getestet.

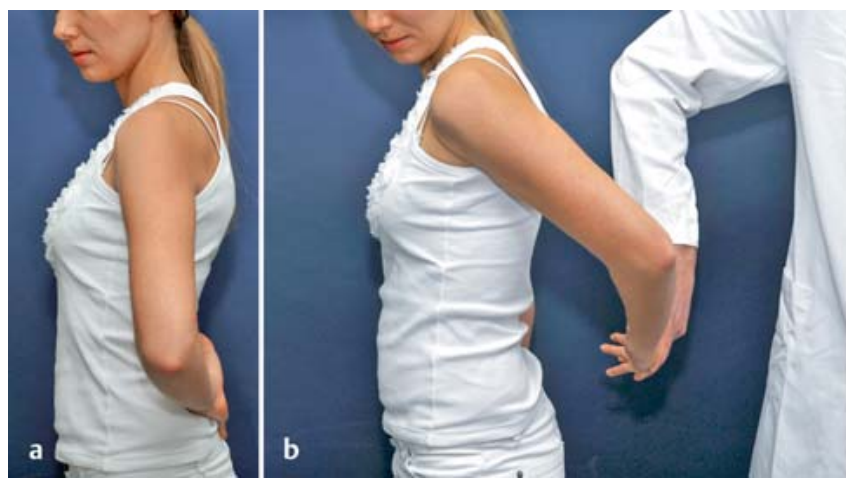

Abb. 9 Lift-off-Test. a Ausgangsposition. b Abheben der Hand vom Körper bei intakter SSC-Funktion.

auf eine Läsion der Rotatorenmanschette kommt eine MRT-Untersuchung zur Anwendung. Während transmurale Rupturen in der nativen MRT gut beurteilt werden können, lassen sich partielle Rupturen in der MR-Arthrografie exakter darstellen [33]. Mittels der MRT kann die Atrophie und fettige Degeneration der Rotatorenmanschette zuverlässig beurteilt werden. Die sonografische Darstellung der Rotatorenmanschette wird von vielen Autoren propagiert. Es wurden Sensitivitäten und Spezifitäten in der Diagnostik von transmuralen Rotatorenmanschettenrupturen von über 90\% beschrieben, bei Partialdefekten von ca. $70-80 \%$ [34,35]. Eine Studie konnte gegenüber der nativen MRT-Diagnostik eine identische und bei Partialläsionen sogar überlegene Wertigkeit der Sonografie nachweisen [36].

\section{M. subscapularis}

Für die Sehne des M. subscapularis (SSC) wurden zahlreiche klinische Tests publiziert. Prinzip der Testung ist jeweils die Innenrotation gegen Widerstand. Von Gerber et al. wurde der Lift-off-Test beschrieben: Der Patient wird dabei aufgefordert, den in der Schürzengriffposition befindlichen Arm gegen den Widerstand des Untersuchers weiter nach dorsal zu bewegen. Der Test gilt als positiv, wenn ein Abheben der Hand vom Körper nicht gelingt (Abb. 9a, b) [37].

Beim Belly-Press-Test befinden sich die Hände des Patienten bei flektiertem Ellenbogen auf dem Bauch. Die Ebene der Ellenbogen befindet sich vor derjenigen des Rumpfes. Aus dieser Stellung heraus wird der Patient gebeten, die Hand mit ganzer Kraft gegen den Bauch zu drücken (Abb.10a). Der Test ist positiv, wenn der Ellenbogen aufgrund des Überwiegens der Außenrotatoren nach dorsal abweicht und dadurch eine Flexion im Handgelenk auftritt (Abb. 10b) [38].

Beim Bear-Hug-Test wird die Handfläche der betroffenen Seite auf die Schulter der Gegenseite gelegt. Das Ellenbogengelenk wird dabei flektiert und vor dem Körper in der Horizontalebene gehalten (Abb. 11a). Der Arm ist somit innenrotiert. Der Untersucher versucht nun den Arm des Patienten in Außenrotation, also senkrecht zum Hebelarm, wegzuziehen. Der Test ist positiv, wenn der Patient nicht in der Lage ist, die Ausgangsposition zu halten (Abb. 11 b) [39].

Zur Testung des Belly-off-Zeichens wird der Arm des Patienten bei $90^{\circ}$ flektiertem Ellenbogen vom Untersucher in eine Flexions- und Innenrotationsstellung ge- 

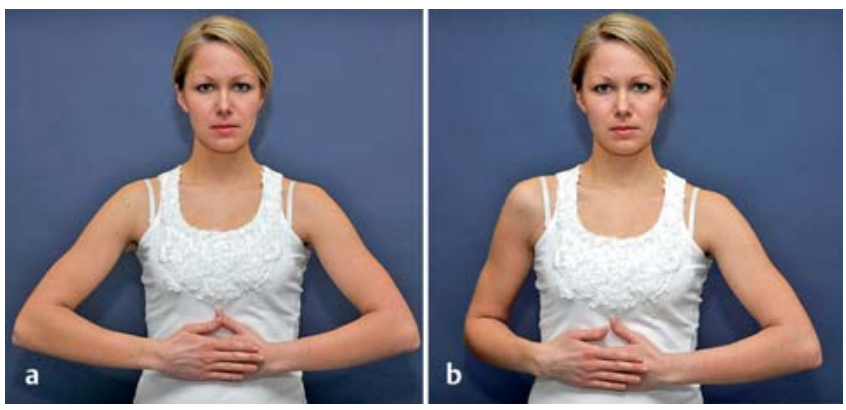

Abb. 10 Belly-Press-Test. a Negativer Test, wenn es gelingt, die Hand auf den Bauch zu drücken und der Ellenbogen dabei vor dem Körper bleibt. Positiver Test bei SSC-Funktionsstörung, wenn dabei der Ellenbogen nach dorsal abweicht und im Handgelenk flektiert wird.
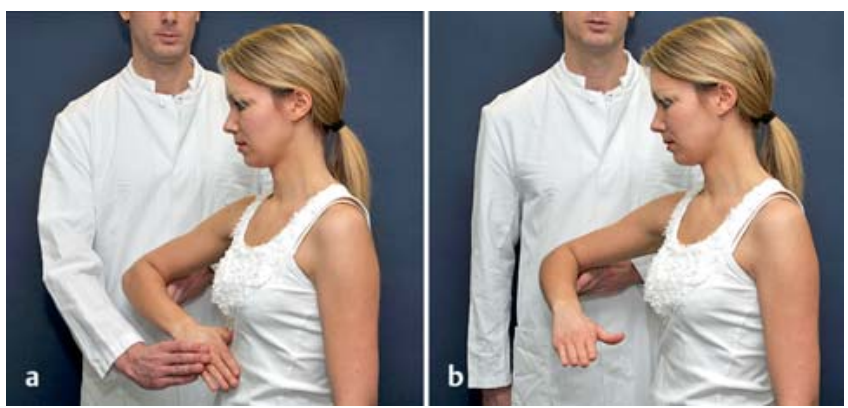

Abb. 12 Belly-off-Zeichen. a Ausgangsposition. b Positiver Test bei SSC-Funktionsstörung, wenn es dem Patienten nicht gelingt, die gestreckte Hand auf dem Bauch zu halten.
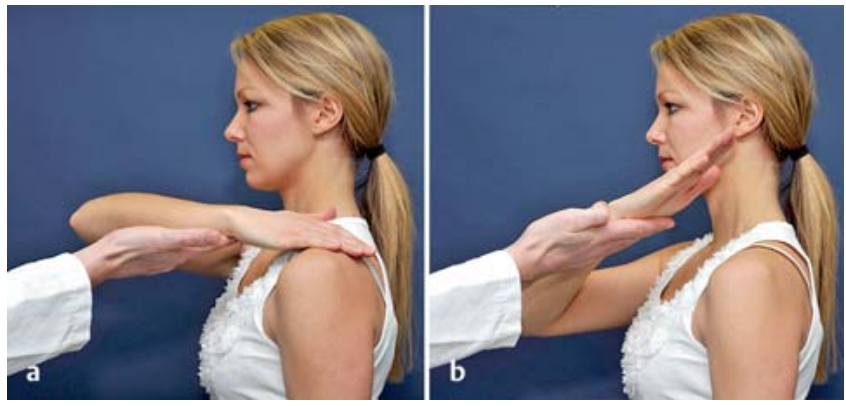

Abb. 11 Bear-Hug-Test. a Ausgangsposition. b Positiver Test bei SSC-Funktionsstörung, wenn es dem Patienten nicht gelingt, den Arm bei Zug des Untersuchers in der Ausgangsposition zu halten.

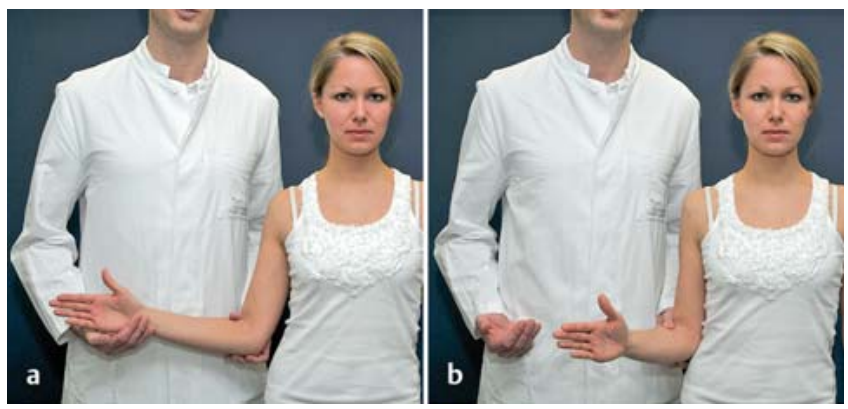

Abb. 13 Außenrotations-Lag-Zeichen. a Ausgangsposition. b Das Zeichen ist positiv, wenn der Patient den Arm nicht in der Position halten kann. Die Differenz wird in Anzahl der Grad notiert. bracht. Dabei wird die Handinnenfläche des Patienten auf dem Bauch des Patienten positioniert (Abb.12a). Anschließend wird der Patient aufgefordert, diese Position zu halten. Der Test ist als positiv zu bewerten, wenn der Patient die gestreckte Hand entweder nicht auf dem Bauch halten kann oder das Handgelenk abknickt (Abb. 12 b) [40].

Verschiedene, u.a. elektromyografische Studien konnten zeigen, dass der SSC bei anliegendem Arm lediglich 50\% der Gesamtinnenrotationskraft erbringt. In Innenrotation steigert sich der Anteil des SSC auf annähernd 90\% [41-43]. Daher werden die meisten Untersuchungstechniken zur Beurteilung des SSC in Innenrotation durchgeführt. Ein Vergleich verschiedener Untersuchungstechniken zeigt, dass der Belly-off-Test mit einer Sensitivität von $87 \%$ und einer Spezifität von $91 \%$ bei einer Genauigkeit von $90 \%$ die höchste Wahrscheinlichkeit bietet, eine SSC-Verletzung klinisch zu detektieren [29]. Einschränkend muss erwähnt werden, dass der Bear-Hug-Test nicht in die Studie eingeschlossen wurde. Für diesen wurde in einer anderen aktuellen Studie ebenfalls eine akzeptable Sensitivität mit $60 \%$ bei einer Spezifität von $92 \%$ beschrieben [39]. Für den in der klinischen Praxis weit verbreiteten Lift-off-
Test und Belly-Press-Test wurden mit einer studienabhängigen Sensitivität von 35-69\% und Spezifität von 48-79\% schlechtere Werte publiziert [17,29,44]. Der Prozentsatz der bei der klinischen Untersuchung trotz Verwendung aller SSC-Tests übersehenen SSC-Läsionen wird in der Literatur mit 15-40\% angegeben [29].

\section{M. infraspinatus/M. teres minor}

Isolierte Läsionen der M. infraspinatus und des $M$. teres minor sind seltener als anteriore Rupturen der Rotatorenmanschette. In der Regel sind sie mit Läsionen des M. supraspinatus vergesellschaftet. Zur Untersuchung der Integrität der Außenrotatoren konnte für den Außenrotationstest in $0^{\circ}$ Abduktion gegen $\mathrm{Wi}$ derstand des Untersuchers eine Sensitivität von $84 \%$ bei einer Spezifität von $53 \%$ gezeigt werden, wenn ein Kraftdefizit vorhanden ist [4]. Lässt sich lediglich ein Schmerz auslösen, ist die Sensitivität erheblich geringer (46\%). In diesen Fällen lässt sich die Sensitivität auf Werte bis $71 \%$ steigern, wenn der Außenrotationstest in $90^{\circ}$ Abduktion in der Skapularebene durchgeführt wird (Patte-Test). Auf diese Weise lässt sich die Krafteinwirkung des M. deltoideus minimieren. Beim Außenrotations-Lag-Zeichen wird der im Ellenbogengelenk $90^{\circ}$ flektierte Arm bei anliegendem Ellenbogen vom Untersucher passiv in die Außenrotation gebracht (Abb.13a). Der Patient wird aufgefordert, diese Position zu halten. Der Test ist positiv, wenn der Arm in der Position nicht gehalten werden kann (Abb. 13b). Castoldi et al. konnten 2009 zeigen, dass das Außenrotations-LagZeichen eine $56 \%$-Sensitivität bei einer 98\%-Spezifität auf eine isolierte Infraspinatussehnenruptur bietet. Bei Beteiligung des M. teres minor stieg die Sensitivität auf $97 \%$. Ebenfalls konnte in dieser Studie ein direkter Zusammenhang zwischen dem Ausmaß der Ruptur und dem Ausmaß des Lag-Zeichens dargestellt werden [45]. Walch et al. konnten bereits 1998 zeigen, dass das sehr ähnlich durchgeführte „Dropping-Arm-Zeichen“ eine 100\%-Sensitivität und -Spezifität für eine höhergradige Verfettung (Goutallier Stadien III und IV) des M. infraspinatus aufweist. Das „Hornblower-Zeichen" ist positiv, wenn der Patient beim Versuch, ein Glas Wasser zu trinken, den Ellenbogen über Handniveau führen muss. Ist das Zeichen positiv, besteht eine 100\%-Sensitivität und eine 93\%Spezifität für eine hochgradige Insuffizienz der Außenrotatoren [46]. 


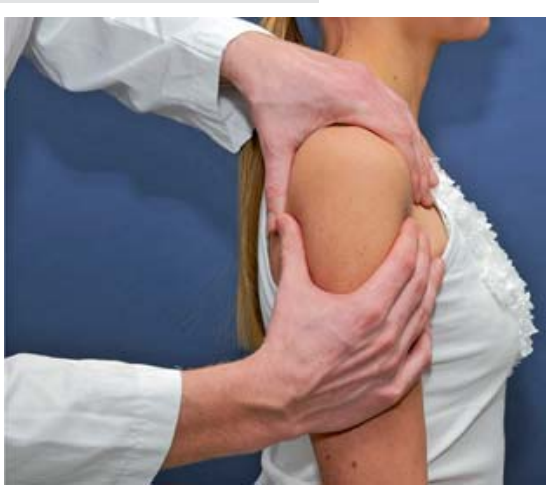

Abb. 14 Schubladentest.

\section{Glenohumerale Instabilitäten}

Speziell bei jungen Menschen mit Schulterschmerzen kann eine Schulterinstabilität die Ursache sein. Wichtig ist hier die Unterscheidung zwischen einer physiologischen und im Regelfall asymptomatischen Laxizität des Glenohumeralgelenks und der pathologischen und meist symptomatischen Instabilität. Ursachen für eine glenohumerale Instabilität können sowohl traumatisch als auch atraumatisch sein.

Die Untersuchung wird mit einer Beurteilung der allgemeinen Laxizität des Weichteilgewebes des Patienten begonnen. Hierzu wird die kontralaterale Schulter untersucht. Zudem werden die Ellenbogengelenke und die Finger auf Überstreckbarkeit getestet. Zur Befunddokumentation eignen sich der Daumenunterarmabstand, der physiologischerweise $6-12 \mathrm{~cm}$ betragen sollte und sich bei hyperlaxen Weichteilverhältnissen auf $0 \mathrm{~cm}$ reduzieren kann.

Die vordere und hintere Translation in der Schulter lässt sich aussagekräftig im Seitenvergleich mit dem vorderen und hinteren Schubladentest am stehenden oder sitzenden Patienten untersuchen (Abb. 14). Je nach Verschieblichkeit erfolgt die Einteilung der Schulterlaxizität nach Hawkins in Grad 0-3 [47]. Der Sulkustest eignet sich zur Beurteilung einer inferioren Instabilität oder generellen Laxizität. Der entspannt herunterhängende Arm des Patienten wird entlang der Körperachse mit axialem Zug nach kaudal gezogen. Bei positivem Test zeigt sich unterhalb des Akromions eine Delle, man spricht vom Sulkuszeichen. Auch hier muss der Test im Seitenvergleich beurteilt werden.

Ein systematischer Review über die Aussagekraft der zur Verfügung stehenden

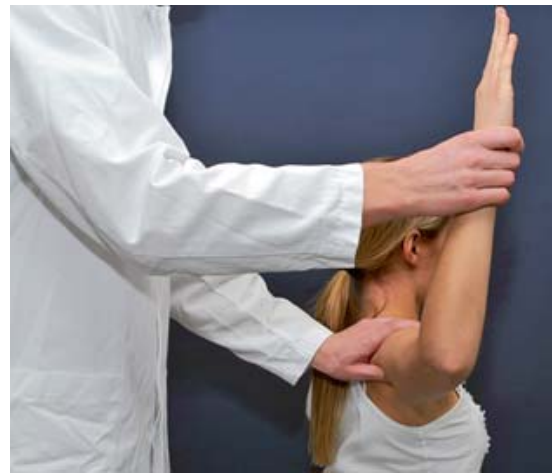

Abb. 15 Apprehension-Test.

klinischen Untersuchungen zeigte, dass sich insbesondere der ApprehensionTest, der Relocationtest und der Surprise-Test eignen, um eine anteriore Instabilität zu diagnostizieren [25]. Beim Apprehension-Test fixiert der Untersucher die Skapula der zu untersuchenden Schulter mit der körpernahen Hand. Der im Ellenbogengelenk $90^{\circ}$ flektierte Arm des Patienten wird in verschiedenen $\mathrm{Ab}$ duktionsgraden um ca. $90^{\circ}$ außenrotiert (Abb. 15). Gleichzeitig übt der Untersucher mit der körpernahen Hand intermittierend einen Druck auf den Humeruskopf von dorsal aus. Der Test gilt als positiv, wenn der Patient als Folge eines Instabilitätsgefühls die Muskulatur anspannt. Die alleinige Angabe von Schmerzen genügt hingegen nicht [48].

Für den Relocation-Test liegt der Patient mit dem Rücken auf einer Untersuchungsliege, der zu untersuchende Arm wird in Abduktion und Außentrotationsstellung geführt, bis ein positives Apprehension-Zeichen entsteht. Der Druck des Untersuchers von ventral rezentriert den Humeruskopf im Glenoid, was vom Patienten als angenehm empfunden wird. Die Außenrotation kann so weiter durchgeführt werden (Abb. 16).

Zur Durchführung des Surprise-Tests hält eine Hand des Untersuchers den Patientenarm in Abduktion und Außenrotation, während die andere Hand des Untersuchers von ventral gegen den $\mathrm{Hu}$ meruskopf drückt und diesen stabilisiert. Bei plötzlichem Lösen des stabilisierenden Druckes kommt es bei positivem Testergebnis zu dem beschriebenen Apprehension-Zeichen [49].

Ein nacheinander durchgeführter Apprehension-Test und Relocation-Test erbringt eine Sensitivität von $81 \%$ und eine Spezifität von 98\% für eine vordere Schulterinstabilität [50]. Die Sensitivität

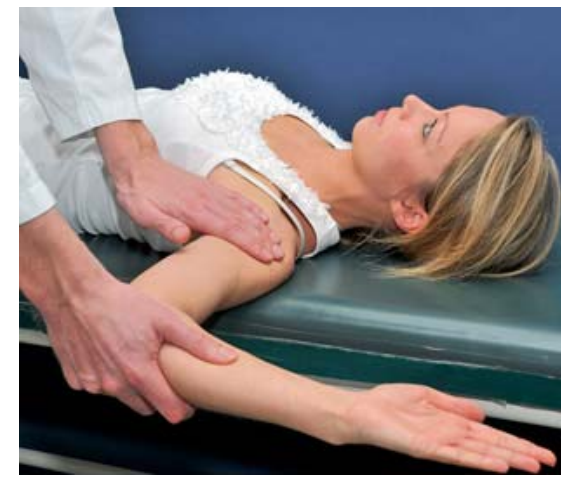

Abb. 16 Relocation-Test.

und Spezifität des Surprise-Tests beträgt $82 \%$ respektive $86 \%$. Bei einem positiven Testergebnis dieser 3 klinischen Tests ist somit eine sehr hohe Wahrscheinlichkeit auf einen anteriore Schultergelenkinstabilität gegeben [51].

Zur Untersuchung einer hinteren Gelenksinstabilität wird der Jerk-Test und der Posterior-Load-Test verwendet. Beim Jerk-Test adduziert und innenrotiert der Untersucher den $90^{\circ}$ flektierten Arm des Patienten progredient. Gleichzeitig wird ein axialer Druck Richtung Glenoid durchgeführt. Bei positivem Testergebnis kann eine dorsale Subluxation ausgelöst werden. Die Rückführung der Bewegung führt zur Reposition (Abb. 17).

Beim Posterior-Load-Test wird der Arm des auf dem Rücken liegenden Patienten in Abduktion und Außenrotation gebracht. Die von ventral kommende 2 . Hand des Untersuchers übt einen nach dorsal gerichteten Druck dorsal gegen den Humeruskopf aus. Dadurch kann ein Schmerz bzw. eine dorsale Subluxation ausgelöst werden. Das Ausmaß der Translation wird mit den Fingern der Hand am Oberarmkopf abgeschätzt (Abb. 18). Für den Jerk-Test zeigte sich, dass bei positivem Test und Schmerzauslösung eine hohe Korrelation mit einem hinteren Labrumschaden bestand [52]. Bei positivem Ergebnis beider Tests besteht eine hohe Wahrscheinlichkeit einer hinteren Gelenksinstabilität.

Eine glenohumerale Instabilität kann in Zusammenschau von Klinik und Anamnese sehr gut beurteilt werden. So konnte gezeigt werden, dass die Aussagefähigkeit der klinischen Untersuchung bezüglich der Diagnosestellung einer BankartLäsion gegenüber der MRT-Untersuchung eine signifikant höhere Sensitivität bei identischer Spezifität hat [53]. Als apparative Diagnostik bieten sich je nach $\mathrm{Ge}$ - 


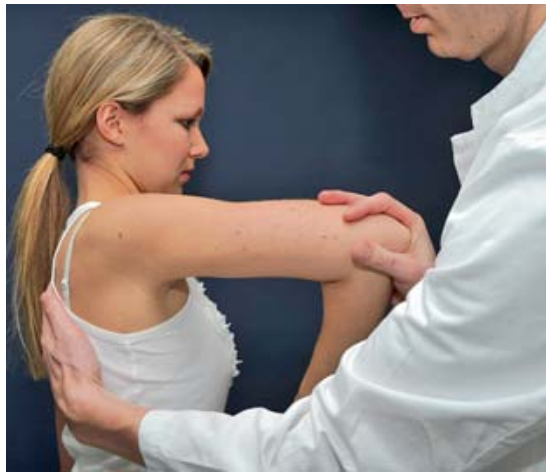

Abb. 17 Jerk-Test.

nese der Instabilität in erster Linie das native MRT, die MR-Arthrografie und die CT-Arthrografie an. Kleine ossäre Bankart-Fragmente können in der nativen MRT oder MR-Arthrografie nur eingeschränkt abgebildet werden, während sie in der CT-Arthrografie gut dargestellt werden können. Dagegen zeigt sich zur Beurteilung kartilaginärer Bankart-Läsionen die MR-Arthrografie der nativen MRT und v.a. gegenüber der CT-Arthrografie überlegen [47]. Sonografisch lassen sich ein Hill-Sachs-Defekt und eine Bankart-Läsion prinzipiell diagnostizieren. Allerdings ist die Ultraschalluntersuchung hinsichtlich Sensitivität und Spezifität dem MRT unterlegen.

\section{Frakturen}

Den Frakturen des Schultergürtels kommt eine erhebliche epidemiologische und ökonomische Bedeutung zu. Klavikulafrakturen sind nach den distalen Radiusfrakturen mit einer Inzidenz von bis zu 15\% die zweithäufigste Fraktur des Erwachsenen, für die proximalen Humerusfrakturen wird aufgrund des demografischen Wandels mit einer Steigerung der Inzidenz um das 3-fache innerhalb der nächsten 2 Jahrzehnte gerechnet [54].

Aufgrund des akuten Traumas mit konsekutiver Schmerzbelastung ist die körperliche Untersuchung des Patienten zumeist initial nur eingeschränkt möglich. Durch Inspektion (Schwellung, Hämatom, Fehlstellung) und Palpation gelingt aber in nahezu allen Fällen bereits eine spezifische Zuordnung der Verletzung, die dann durch eine fokussierte Bildgebung weiter abgeklärt werden kann.

Eine besondere Bedeutung der klinischen Untersuchung kommt der Erkennung möglicher Begleitverletzungen zu. So müssen bei Klavikulafrakturen im

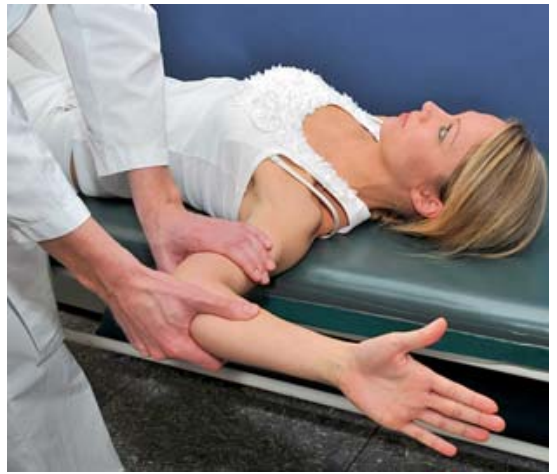

Abb. 18 Posterior-Load-Test.

Rahmen von Hochrasanztraumata Begleitverletzungen von Rippen, Skapula und Halswirbelsäule ausgeschlossen werden. Bei proximalen Humerusfrakturen ist die Funktionsprüfung des N. axillaris obligat. Bei Skapulafrakturen ist die gezielte Funktionsüberprüfung des N. suprascapularis und des N. axillaris essenziell.

Bei allen Frakturen wird die periphere Durchblutung, Motorik und Sensibilität im Seitenvergleich beurteilt und dokumentiert.

Empfohlene apparative Diagnostik:

- Klavikulafraktur: konventionelle Röntgenaufnahme Klavikula a.-p. und schräg sowie Schulter axial.

- proximale Humerusfraktur: konventionelle Röntgenaufnahme Schulter a.-p., outlet und axial. Bei intraartikulärer Fraktur und/oder komplexer Frakturmorphologie wird in unserer Klinik eine Computertomografie der Schulter durchgeführt.

- Skapulafrakturen: konventionelle Röntgenaufnahme der Schulter a.-p., axial und transskapulär. Ggf. konventionell radiologische Zusatzaufnahme zur Beurteilung von Akromion- oder Glenoidfrakturen (Westpoint-Aufnahme) bzw. zur Beurteilung von Korakoidfrakturen (Stryker-Aufnahme). In unserer Klinik wird zur Beurteilung der Frakturmorphologie eine Computertomografie der Schulter mit 3-DRekonstruktionen durchgeführt.

\section{Fazit}

Die Palette möglicher Untersuchungstechniken der Schulter ist schwer überschaubar. Eine Konzentration auf wenige, dafür aber aussagekräftige Tests ist daher sinnvoll. Sinnvoll ist es daher, sich ein Portfolio von Tests zurechtzulegen und einen standardisierten Unter- suchungsablauf durchzuführen. Die Beschränkung auf möglichst wenige, dafür aber aussagekräftige Tests lässt in den meisten Fällen bereits klinisch eine Diagnosestellung zu. Die Verdachtsdiagnose sollte dann mit der sinnvollen apparativen Diagnostik ergänzt werden.

Dieser klinische und apparative Untersuchungsalgorithmus sollte von allen Mitarbeitern einer Abteilung sicher beherrscht werden, um eine vergleichund reproduzierbare Beurteilung von Schulterpathologien zu erreichen. Ebenso sollten standardmäßig Scores und der aktive wie passive Bewegungsumfang erhoben werden.

\section{Literatur}

${ }^{1}$ Murrell GA, Walton JR. Diagnosis of rotator cuff tears. Lancet 2001; 357: 769-770

2 Walton J et al. Diagnostic values of tests for acromioclavicular joint pain. J Bone Joint Surg Am 2004; 86-A: 807-812

${ }^{3}$ Gerber C, Galantay RV, Hersche 0 . The pattern of pain produced by irritation of the acromioclavicular joint and the subacromial space. J Shoulder Elbow Surg 1998; 7: 352-355

${ }^{4}$ Itoi $E$ et al. Are pain location and physical examinations useful in locating a tear site of the rotator cuff? Am J Sports Med 2006; 34: 256 264

${ }^{5}$ Scheibel M, Habermeyer P. [Current procedures for clinical evaluation of the shoulder] Orthopade 2005; 34: 267-283

${ }^{6}$ Terwee CB et al. Interobserver reproducibility of the visual estimation of range of motion of the shoulder. Arch Phys Med Rehabil 2005; 86: 1356-1361

7 Kolber MJ et al. The reliability and concurrent validity of scapular plane shoulder elevation measurements using a digital inclinometer and goniometer. Physiother Theory Pract 2012; 28: 161-168

8 Constant CR, Murley AH. A clinical method of functional assessment of the shoulder. Clin Orthop Relat Res 1987; 214: 160-164

${ }^{9}$ Lundberg BJ. The frozen shoulder. Clinical and radiographical observations. The effect of manipulation under general anesthesia. Structure and glycosaminoglycan content of the joint capsule. Local bone metabolism. Acta Orthop Scand Suppl 1969; 119: 1-59

${ }_{10}$ Reeves $B$. The natural history of the frozen shoulder syndrome. Scand J Rheumatol 1975; 4: 193-196

${ }^{11}$ Kirkley A, Griffin S, Dainty K. Scoring systems for the functional assessment of the shoulder. Arthroscopy 2003; 19: 1109-1120

12 Conboy VB et al. An evaluation of the Constant-Murley shoulder assessment. J Bone Joint Surg Br 1996; 78: 229-232

13 Gilbart MK Gerber C. Comparison of the subjective shoulder value and the Constant score. J Shoulder Elbow Surg 2007; 16: $717-$ 721

${ }^{14}$ Michener LA et al. Reliability and diagnostic accuracy of 5 physical examination tests and combination of tests for subacromial impingement. Arch Phys Med Rehabil 2009; 90: 1898-1903

${ }^{15}$ Fodor $D$ et al. Shoulder impingement syndrome: correlations between clinical tests and ultrasonographic findings. Ortop Traumatol Rehabil 2009; 11: 120-126 
16 Silva L et al. Accuracy of physical examination in subacromial impingement syndrome. Rheumatology (Oxford) 2008; 47: 679-683

17 Salaffi $F$ et al. Clinical value of single versus composite provocative clinical tests in the assessment of painful shoulder. J Clin Rheumatol 2010; 16: 105-108

18 Park HB et al. Diagnostic accuracy of clinical tests for the different degrees of subacromial impingement syndrome. J Bone Joint Surg Am 2005; 87: 1446-1455

19 O'Brien SJ et al. The active compression test: a new and effective test for diagnosing labra tears and acromioclavicular joint abnormality. Am J Sports Med 1998; 26: 610-613

${ }^{20}$ Ebinger $N$ et al. A new SLAP test: the supine flexion resistance test. Arthroscopy 2008; 24: 500-505

21 Fowler EM, Horsley IG, Rolf CG. Clinical and arthroscopic findings in recreationally active patients. Sports Med Arthrosc Rehabil Ther Technol 2010; 2: 2

22 Bencardino JT et al. Superior labrum anteriorposterior lesions: diagnosis with MR arthrography of the shoulder. Radiology 2000; 214 : 267-271

23 Palmer WE, Brown JH, Rosenthal DI. Rotator cuff: evaluation with fat-suppressed MR arthrography. Radiology 1993; 188: 683-687

24 Gill HS et al. Physical examination for partial tears of the biceps tendon. Am J Sports Med 2007; 35: 1334-1340

25 Hegedus EJ et al. Which physical examination tests provide clinicians with the most value when examining the shoulder? Update of a systematic review with meta-analysis of individual tests. Br J Sports Med 2012; 46: 964-978

26 Zanetti $M$ et al. Tendinopathy and rupture of the tendon of the long head of the biceps brachii muscle: evaluation with MR arthrography. AJR Am J Roentgenol 1998; 170: 15571561

27 Hedtmann A. Weichteilerkrankungen der Schulter - Subakromialsyndrome. Orthopädie und Unfallchirurgie up2date 2009; 4 85-103

28 Yamaguchi $K$ et al. The demographic and morphological features of rotator cuff disease. A comparison of asymptomatic and symptomatic shoulders. J Bone Joint Surg Am 2006; 88: 1699-1704

29 Bartsch $M$ et al. Diagnostic values of clinical tests for subscapularis lesions. Knee Surg Sports Traumatol Arthrosc 2010; 18: 17121717
30 Scheibel M, Habermeyer P. Klinische Diagnostik von Rotatorenmanschettenläsionen. Arthroskopie 2007; 20: 6-12

31 Jobe FW, Moynes DR. Delineation of diagnostic criteria and a rehabilitation program for rotator cuff injuries. Am J Sports Med 1982; 10: 336-339

${ }^{32} \mathrm{Kim} \mathrm{E}$ et al. Interpreting positive signs of the supraspinatus test in screening for torn rotator cuff. Acta Med Okayama 2006; 60: 223228

33 Hodler J et al. Rotator cuff disease: assessment with MR arthrography versus standard MR imaging in 36 patients with arthroscopic confirmation. Radiology 1992; 182: 431-436

${ }^{34}$ Hedtmann A, Fett $H$. [Ultrasonography of the shoulder in subacromial syndromes with disorders and injuries of the rotator cuff]. Orthopade 1995 ; 24 : 498-508

35 Teefey SA et al. Detection and measurement of rotator cuff tears with sonography: analysis of diagnostic errors. AJR Am J Roentgenol 2005; 184: 1768-1773

36 Dinnes J et al. The effectiveness of diagnostic tests for the assessment of shoulder pain due to soft tissue disorders: a systematic review. Health Technol Assess 2003; 7: 1-166

37 Gerber C, Krushell RJ. Isolated rupture of the tendon of the subscapularis muscle. Clinical features in 16 cases. J Bone Joint Surg $\mathrm{Br}$ 1991; 73: 389-394

38 Gerber $C$, Hersche O, Farron A. Isolated rupture of the subscapularis tendon. J Bone Joint Surg Am 1996; 78: 1015-1023

39 Barth JR, Burkhart SS, De Beer JF. The bear-hug test: a new and sensitive test for diagnosing a subscapularis tear. Arthroscopy 2006; 22: 1076-1084

40 Scheibel $M$ et al. The belly-off sign: a new clinical diagnostic sign for subscapularis lesions. Arthroscopy 2005; 21: 1229-1235

41 Greis PE et al. Validation of the lift-off test and analysis of subscapularis activity during maximal internal rotation. Am J Sports Med 1996: 24 : 589-593

42 Kuechle DK et al. The relevance of the moment arm of shoulder muscles with respect to axial rotation of the glenohumeral joint in four positions. Clin Biomech (Bristol, Avon) 2000; 15: 322-329

43 Veeger HE et al. Inertia and muscle contraction parameters for musculoskeletal modelling of the shoulder mechanism. J Biomech 1991; 24: 615-629

${ }^{44} \mathrm{Kim}$ HA, Kim SH, Seo YI. Ultrasonographic findings of the shoulder in patients with rheumatoid arthritis and comparison with physical examination. J Korean Med Sci 2007; 22: 660-666

45 Castoldi F, Blonna D, Hertel R. External rotation lag sign revisited: accuracy for diagnosis of full thickness supraspinatus tear. J Shoulder Elbow Surg 2009; 18: 529-534

46 Walch $G$ et al. The 'dropping' and 'hornblower's' signs in evaluation of rotator-cuff tears. J Bone Joint Surg Br 1998; 80: 624-628

47 Habermeyer P. Schulterchirurgie. 4. Aufl München: Urban \& Fischer/Elsevier GmbH; 2010: 155-160

48 Rowe CR, Zarins B. Recurrent transient subluxation of the shoulder. J Bone Joint Surg Am 1981; 63: 863-872

49 Gross ML, Distefano MC. Anterior release test. A new test for occult shoulder instability. Clin Orthop Relat Res 1997; 339: 105-108

50 Farber AJ et al. Clinical assessment of three common tests for traumatic anterior shoulder instability. J Bone Joint Surg Am 2006; 88: 1467-1474

51 Lo IK et al. An evaluation of the apprehension, relocation, and surprise tests for anterior shoulder instability. Am J Sports Med 2004; 32: 301-307

$52 \mathrm{Kim} \mathrm{SH}$ et al. Painful jerk test: a predictor of success in nonoperative treatment of posteroinferior instability of the shoulder. Am J Sports Med 2004; 32: 1849-1855

${ }^{53}$ Liu SH et al. Diagnosis of glenoid labral tears. A comparison between magnetic resonance imaging and clinical examinations. Am J Sports Med 1996; 24: 149-154

54 Voigt C, Katthagen C, Lill $H$. Frakturen des Schultergürtels. Orthopädie und Unfallchirurgie up2date 2009; 4: 321-335

\section{Dr. med. Uli Schmiddem \\ Assistenzarzt \\ Univ.-Prof. Dr. med. C. Krettek}

Ärztlicher Direktor

Priv.-Doz. Dr. med. Rupert Meller

Medizinische Hochschule Hannover

Unfallchir. Klinik

Carl-Neuberg-Straße 1

30625 Hannover

Schulter@mh.hannover.de

rupertmeller@hotmail.com 